\title{
Polymer Scaffolds for Small-Diameter Vascular Tissue Engineering
}

\author{
By Haiyun Ma, Jiang Hu, and Peter X. Ma*
}

To better engineer small-diameter blood vessels, a few types of novel scaffolds are fabricated from biodegradable poly(L-lactic acid) (PLLA) by means of thermally induced phase-separation (TIPS) techniques. By utilizing the differences in thermal conductivities of the mold materials and using benzene as the solvent scaffolds with oriented gradient microtubular structures in the axial or radial direction can be created. The porosity, tubular size, and the orientational direction of the microtubules can be controlled by the polymer concentration, the TIPS temperature, and by utilizing materials of different thermal conductivities. These gradient microtubular structures facilitate cell seeding and mass transfer for cell growth and function. Nanofibrous scaffolds with an oriented and interconnected microtubular pore network are also developed by a one-step TIPS method using a benzene/tetrahydrofuran mixture as the solvent without the need for porogen materials. The structural features of such scaffolds can be conveniently adjusted by varying the solvent ratio, phase-separation temperature, and polymer concentration to mimic the nanofibrous features of an extracellular matrix. These scaffolds were fabricated for the tissue engineering of small-diameter blood vessels by utilizing their advantageous structural features to facilitate blood-vessel regeneration.

are not natural tissues. For small-diameter (inner diameter $<6 \mathrm{~mm}$ ) blood vessels (SDBV), poor patency because of thrombosis and hyperplasia is a major problem when Dacron and PTFE are used. Tissueengineered living blood vessels could potentially serve as a better source of grafts for patients. ${ }^{[2-4]}$ Scaffolds play a pivotal role in tissue engineering. ${ }^{[5-11]}$ Since Shinoka, $\mathrm{Ma}$, and colleagues reported the feasibility of constructing heart-valve leaflets and blood vessels using synthetic biodegradable polymer scaffolds, ${ }^{[12-15]}$ there has been a lot of very active research on vascular tissue engineering. ${ }^{[16-31]}$ By using synthetic biodegradable polymer scaffolding, it has been demonstrated that tissue-engineered blood-vessel substitutes could achieve adequate mechanical strength for arterial implantation either directly ${ }^{[13]}$ or after invitro pre-cultivation. ${ }^{[30]}$

The structural design of the biodegradable synthetic matrices or scaffolds is a key issue in engineering living vascular substi-

\section{Introduction}

Cardiovascular disease is still the leading cause of mortality in the United States, with 1 million lives lost each year and healthcare-related costs exceeding 300 billion dollars. ${ }^{[1]}$ Largediameter (inner diameter $>6 \mathrm{~mm}$ ) blood vessels have been successfully replaced with non-degradable polymeric materials such as Dacron (polyethylene terephthalate, PET) and expanded polytetrafluoroethylene (ePTFE). However, Dacron and PTFE are not the ideal solution for replacing blood vessels because they

[*] Dr. H. Ma, Dr. J. Hu, Prof. P. X. Ma

Department of Biologic and Materials Sciences

1011 North University Ave., Room 2211

The University of Michigan

Ann Arbor, MI 48109-1078 (USA)

E-mail:mapx@umich.edu

Prof. P. X. Ma

Department of Biomedical Engineering

University of Michigan

Ann Arbor, MI, 48109 (USA)

Prof. P. X. Ma

Macromolecular Science and Engineering Center

University of Michigan

Ann Arbor, MI, 48109 (USA) tutes, especially for SDBVs. To fabricate the blood-vessel scaffolds, several factors should be considered: 1) biocompatibility and biodegradability of the polymers, 2) a high porosity with a structure suitable for cell seeding, distribution, function, and tissue regeneration, 3) structural similarity to the native extracellular matrix (ECM) - such as the tubular geometry at a macroscopic level and the fibrous architecture at the nanometer scale, and 4) adequate mechanical properties to support tissue regeneration. Although there are many ongoing efforts to develop scaffolds for blood-vessel tissue engineering, the scaffolds developed up to this point have various drawbacks and limitations. Yang fabricated scaffolds for SDBV tissue engineering using biodegradable polymers. ${ }^{[32]}$ However, the micropores in these scaffolds were randomly oriented and with non-fibrous walls, thus, having a low structural similarity to blood vessels. Our lab developed the directional thermally induced phase-separation (TIPS) technique to fabricate biodegradable polymer scaffolds consisting of an oriented array of open microtubules. ${ }^{[33]}$ Wang and colleagues ${ }^{[34]}$ prepared poly(lactic-co-glycolic acid) (PLGA) blood-vessel scaffolds by adopting this method to achieve an axially orientated pore structure in the tubular walls. However, these scaffolds had solid walls without nanofibrous structure and had very low degree of lateral interconnectivity. Vaz ${ }^{[35]}$ developed a bi-layered tubular scaffold composed of a stiff and oriented PLA outer shell and a random polycaprolactone (PCL)

DOI: 10.1002/adfm.201000922 
fibrous inner layer (PLA/PCL) using the electrospinning technique. A major limitation of such a scaffold was that the pores were too small to allow uniform cell seeding and cultivation. Our laboratory developed a phase-separation technique to fabricate synthetic nanofibrous matrices ${ }^{[36]}$ as well as techniques to generate interconnected spherical pores within the polymer scaffolds. ${ }^{[33,37,38]}$ However, this is the first time that these techniques are used for the fabrication of tubular scaffolds with oriented pores to facilitate blood-vessel tissue engineering.

In this work, we aimed to develop novel scaffolds with structural features on multiple scales to mimic certain key structural features of the extracellular matrix (ECM) and with designed pores/channels to facilitate cell seeding and three-dimensional blood-vessel regeneration.

\section{Results}

\subsection{Architecture}

When the temperature of a polymer solution is reduced below the freezing point of the solvent, solid-liquid phase separation occurs and a porous scaffold can be obtained after removal of the solidified solvent. ${ }^{[1]}$ When a typical mold such as a Teflon vial is used, random pores are generated as previously reported. ${ }^{[11]}$ These randomly arranged pores are not ideal, however, for uniform cell seeding, subsequent proliferation, and organized blood-vessel regeneration. In the present work, the different parts of the molds are made of different materials (e.g., thermally more conductive steel and less conductive Teflon) so that the different thermal conductivities of these materials are utilized to regulate the phase-separation process (Figure 1). When such a mold is cooled, the phase separation of the polymer solution inside occurs under a directionally distributed temperature field because of the different thermal conductivities of the materials. For example, the steel parts reach lower temperatures (such as the freezing point of the solvent) more rapidly than the Teflon parts. Thus, temperature gradients are


Figure 1. View of the molds used for preparing the oriented gradient microtubule-structured scaffolds: (left) schematic illustrations of the molds; (top right) appearance of the molds with two different materials; (bottom right) appearance of produced scaffolds.

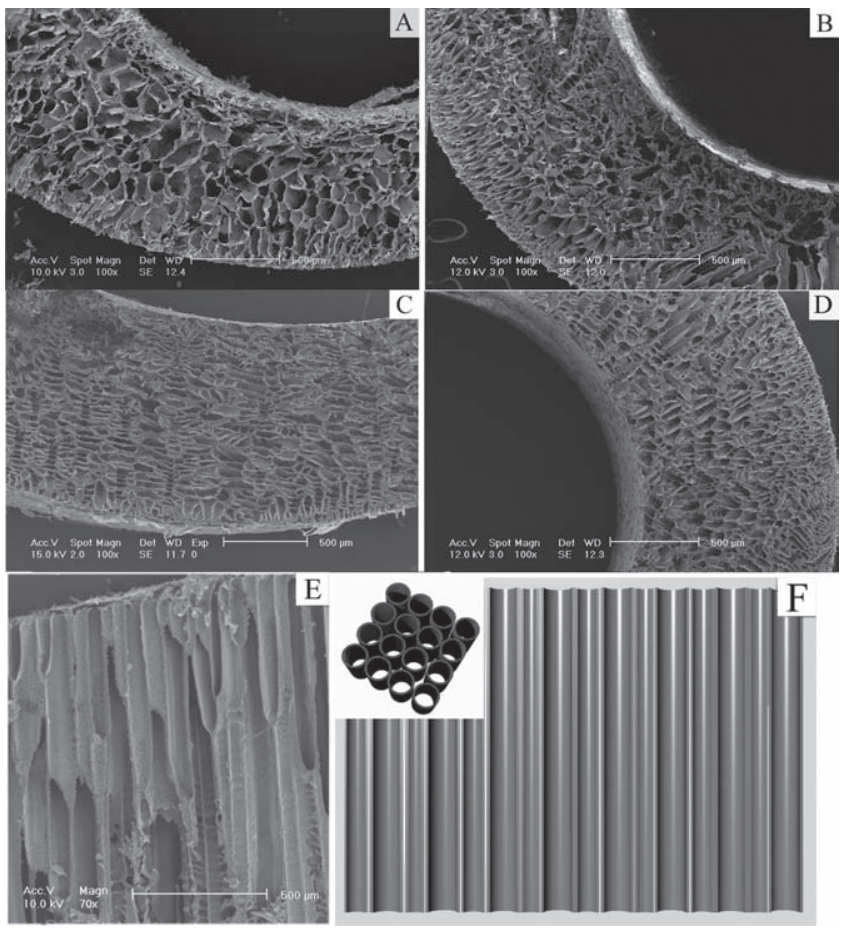

Figure 2. A-D) Scanning electron microscopy (SEM) images of the cross section of the microtubules formed under an axial temperature gradient at $-20{ }^{\circ} \mathrm{C}$ for PLLA/benzene solutions with different concentrations $(\mathrm{w} / \mathrm{v})$ : A) $2.5 \%$; B) $5.0 \%$; C) $7.5 \%$; D) $10.0 \%$. E) SEM image of the longitudinal section, $7.5 \%$. F) Illustration of the microtubular structure (inset - cross section).

formed in the mold, leading to directional phase separation and the formation of oriented structures. Detailed structural features and the physical properties of these structures are discussed below.

\subsubsection{Microtubular Gradient Structure}

Axial oriented structure: When a polymer solution was placed in a mold consisting of a steel bottom and Teflon wall/shaft/top, a temperature gradient was formed from bottom to top (from low to high) and maintained uniaxially during the thermally induced phase-separation process. The characteristic architecture of an array of parallel microtubules was achieved (Figure 2). When benzene was used as the solvent of poly(L-lactic acid) (PLLA), the cross sections of the microtubules were polygons with 3-7 sides. When the polymer concentration was increased from $2.5 \%$ to $10 \%$, the porosity of the formed scaffolds was slightly reduced from $95 \%$ to $90 \%$, and the average pore size was also decreased from 120-150 $\mu \mathrm{m}$ to $80-120 \mu \mathrm{m}$. When the phase-separation temperature was decreased from $-20{ }^{\circ} \mathrm{C}$ to $-196{ }^{\circ} \mathrm{C}$ (liquid nitrogen), the pore size was greatly decreased from $115-140 \mu \mathrm{m}$ to $20-40 \mu \mathrm{m}$ (Table 1 ).

Radially oriented gradient structure: When the mold was composed of a steel wall and Teflon bottom/top/shaft or a reversed composition (steel shaft/bottom/top and a Teflon wall), a temperature gradient was formed in the radial direction either from the outside (warmer) to the inside (colder) or from the inside (warmer) to the outside (colder). Accordingly, radially oriented 
www.MaterialsViews.com

Table 1. Structural and mechanical properties of the solid-walled PLLA scaffolds.

\begin{tabular}{|c|c|c|c|c|c|c|c|c|c|c|c|}
\hline \multirow[t]{2}{*}{ Polymer } & \multirow[t]{2}{*}{ Solvent } & \multirow[t]{2}{*}{$\begin{array}{l}\text { Concentration } \\
\text { (w/v, [\%]) }\end{array}$} & \multirow[t]{2}{*}{$\begin{array}{l}\text { Phase separation } \\
\text { temperature }\left[{ }^{\circ} \mathrm{C}\right]\end{array}$} & \multirow[t]{2}{*}{$\begin{array}{c}\text { Pore } \\
\text { structure }\end{array}$} & \multirow[t]{2}{*}{$\begin{array}{l}\text { Density } \\
{\left[\mathrm{g} \mathrm{cm}^{-3}\right]}\end{array}$} & \multirow[t]{2}{*}{ Porosity [\%] } & \multirow[t]{2}{*}{ Pore Size $[\mu \mathrm{M}]$} & \multicolumn{2}{|c|}{$\begin{array}{c}\text { Compressive modulus } \\
{[\mathrm{MPa}]}\end{array}$} & \multicolumn{2}{|c|}{$\begin{array}{c}\text { Compressive yield } \\
\text { strength [MPa] }\end{array}$} \\
\hline & & & & & & & & axial & radial & axial & radial \\
\hline PLLA & Benzene & 2.5 & -20 & 4 & 0.064 & 94.9 & $0-200$ & - & - & - & - \\
\hline PLLA & Benzene & 2.5 & -20 & 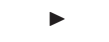 & 0.063 & 95.0 & $0-210$ & - & - & - & - \\
\hline PLLA & Benzene & 2.5 & -20 & $\Delta$ & 0.065 & 94.8 & $120-150$ & 0.55 & 0.32 & 0.22 & 0.13 \\
\hline PLLA & Benzene & 5.0 & -20 & 4 & 0.098 & 92.2 & $0-150$ & 1.7 & 4.9 & 0.16 & 0.40 \\
\hline PLLA & Benzene & 5.0 & $\mathrm{LN}$ & 4 & 0.096 & 92.4 & $10-20$ & 2.1 & 5.2 & 0.20 & 0.45 \\
\hline PLLA & Benzene & 5.0 & -20 & $\triangleright$ & 0.097 & 92.3 & $0-155$ & 1.8 & 4.5 & 0.17 & 0.43 \\
\hline PLLA & Benzene & 5.0 & $\mathrm{LN}$ & $\triangleright$ & 0.094 & 92.5 & $10-20$ & 2.0 & 5.0 & 0.21 & 0.48 \\
\hline PLLA & Benzene & 5.0 & -20 & $\Delta$ & 0.102 & 91.9 & $115-140$ & 3.8 & 2.0 & 0.38 & 0.15 \\
\hline PLLA & Benzene & 5.0 & $\mathrm{LN}$ & $\Delta$ & 0.101 & 92.0 & $20-40$ & 4.2 & 2.9 & 0.43 & 0.23 \\
\hline PLLA & Benzene & 7.5 & -20 & 4 & 0.123 & 90.2 & $0-120$ & 2.6 & 6.3 & 0.27 & 0.53 \\
\hline PLLA & Benzene & 7.5 & -20 & $>$ & 0.125 & 90.1 & $0-120$ & 2.5 & 5.9 & 0.24 & 0.56 \\
\hline PLLA & Benzene & 7.5 & -20 & $\Delta$ & 0.125 & 90.1 & $90-130$ & 4.5 & 2.8 & 0.44 & 0.21 \\
\hline PLLA & Benzene & 10.0 & -20 & 4 & 0.133 & 89.4 & $0-100$ & 3.6 & 8.4 & 0.37 & 0.85 \\
\hline PLLA & Benzene & 10.0 & -20 & $\triangleright$ & 0.135 & 89.3 & $0-100$ & 3.9 & 8.7 & 0.40 & 0.88 \\
\hline PLLA & Benzene & 10.0 & -20 & $\Delta$ & 0.131 & 89.6 & $80-120$ & 7.7 & 4.0 & 0.68 & 0.34 \\
\hline
\end{tabular}

४: O/I oriented structure; $\boldsymbol{\nabla}$ : / O oriented structure; $\mathbf{\Lambda}$ : bottom-top oriented structure

gradient pore structures of the PLLA scaffolds were formed (Figure 3-5). They were named O/I (microtube size decreased gradually from outside wall to inside wall) and I/O (microtube size decreased gradually from inside wall to outside wall) structures in the following discussion.

It was found that the resulting scaffolds had an oriented fishbone-like architecture, which had parallel microtubes with thin partitions. Also, the microtube diameters became radially larger or smaller along the direction of the temperature gradient (summarized in Figure 6). For instance, the microtube size of the I/O structured gradient scaffold was gradually reduced from $200 \mu \mathrm{m}$ to nearly zero, whereas that of the $\mathrm{O} / \mathrm{I}$ structured scaffold was gradually increased from about 30 to $200 \mu \mathrm{m}$. The microtube size was reduced with increasing polymer concentration (Figure 2-5 and Table 1 ). The radial temperature-gradient direction did not significantly affect the average pore size at the same polymer concentration. The pore size formed under the radial temperature gradient was also greatly reduced from $200 \mu \mathrm{m}$ to $20 \mu \mathrm{m}$ when the temperature was changed from $-20{ }^{\circ} \mathrm{C}$ to $-196{ }^{\circ} \mathrm{C}$ (Figure $3-5$ and Table 1). The phase-separation temperature had no obvious effect on the porosity (Table 1). It is worth noting that there was no obvious gradient microtubule structure and no fishbone-like structure at the phase-separation temperature of $-196{ }^{\circ} \mathrm{C}$ (Figure 5).

The phase-separation temperature and the direction of the temperature gradient both had clear effects on the micro-architecture of the PLLA scaffolds. This is related to the nature of the solid-liquid phase separation, in other words, to the crystallization of the solvent, which controls the pore architecture of the formed scaffold. ${ }^{[33]}$ Different directions of the temperature gradient resulted in different crystallization paths of the solvent (benzene). Benzene has a shorter

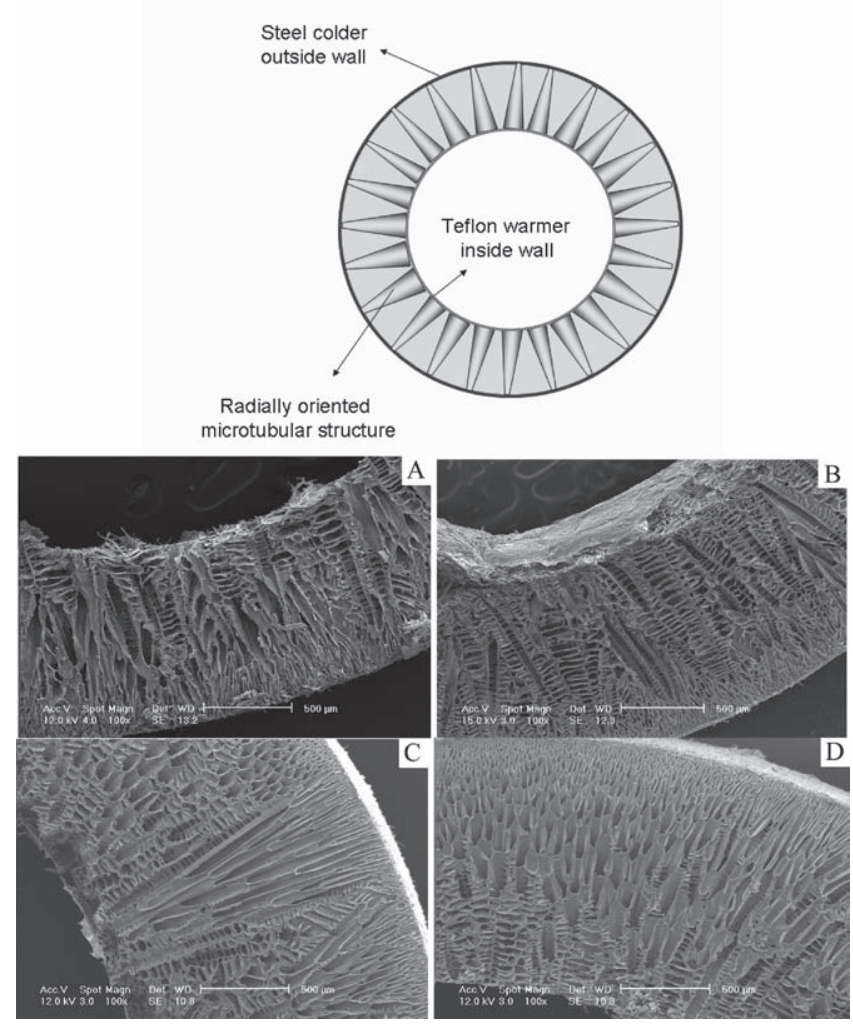

Figure 3. SEM images of cross sections of a vessel scaffold prepared under a radial temperature gradient $\left(\mathrm{I} / \mathrm{O}\right.$ structure) at $-20^{\circ} \mathrm{C}$ for PLLA/ benzene solutions with different concentrations (w/v): A) 2.5\%; B) $5.0 \%$; C) $7.5 \%$; D) $10.0 \%$. 
Teflon warmer outside wall



Figure 4. SEM images of cross sections of vessel scaffolds prepared under a radial temperature gradient $\left(\mathrm{O} / \mathrm{I}\right.$ structure) at $-20^{\circ} \mathrm{C}$ from PLLA/ benzene solutions with different concentrations (w/v): A) 2.5\%; B) $5.0 \%$; C) $7.5 \%$; D) $10.0 \%$

crystallization path under the radial temperature gradient as compared to under the axial temperature gradient because of the thinner walls. Therefore, at extremely low temperatures $\left(-196^{\circ} \mathrm{C}\right)$ benzene rapidly crystallized and there was likely not enough time to form the gradient and branched pore structure.

\subsubsection{Nanofibrous Interconnected Structure}

It has been shown in our previous studies that the nanofibrous architecture advantageously enhances bone and cartilage tissue regeneration. ${ }^{[37,39-43]}$ Natural blood vessels are largely composed of collagens Type I and III and elastin, which are fibers of nanometer dimensions. Therefore, using scaffolds that mimic nanosized fiber architecture may be advantageous to engineer blood vessels.

Although the scaffolds resulting from PLLA/benzene solutions are partly similar to natural blood vessels in structure, their solid pore walls are greatly different from blood vessels that are composed of nanofibrous proteins. In the following, a benzene/tetrahydrofuran (THF) mixture was used as the solvent for PLLA in the TIPS procedure to create a nanofibrous scaffold with oriented and interconnected microtubules (Figure 7). When the solvent was THF alone, the scaffold was composed of nanofibers, but contained few micrometer-sized pores (Figure $7 \mathrm{~J}, \mathrm{~K}$ ). However, when benzene and THF were mixed at various ratios, nanofibrous matrices with oriented microtubules on the micrometer scale were created. Similar to the scaffold made from a PLLA/benzene solution (see section 2.1.1), these scaffolds had low densities and high porosities. The density increased and the porosity decreased with increasing polymer concentration. For a benzene/THF ratio of 9:1 (v/v), the diameter of the microtubule ranged from about $80 \mu \mathrm{m}$ to $250 \mu \mathrm{m}$. A partly nanofibrous structure was formed in these microtubule walls and the average diameter of the nanofibers was $165 \pm 15 \mathrm{~nm}$. When the ratio of benzene/THF was $8: 2(\mathrm{v} / \mathrm{v})$ and $6: 4(\mathrm{v} / \mathrm{v})$, a complete nanofibrous structure was achieved. The average fiber diameter of the fibrous matrices did not change statistically with the concentration of the polymer or the ratio of benzene/THF. The fiber diameter was similar to that obtained for nanofibrous PLLA matrices fabricated using THF alone. However, the size of the microtubules was greatly reduced with a further increase of the THF percentage in the solvent mixture.

At the same benzene/THF ratio, the pore size decreased with decreasing temperature (Table 2) and increasing polymer concentration (Figure 8 and Table 2), although the diameter of the nanofibers did not change appreciably with the temperature (Figure 9 and Table 2). It is important to note that in the benzene/THF ratio range studied, the microtubules with nanofibrous walls were highly interconnected "tunnels" in the scaffolds, and the "tunnels" were also oriented in the orthogonal directions. Table 2 summarizes the structural properties of the matrices described here.

Table 2. Structural and mechanical properties of the nanofibrous PLLA scaffolds.

\begin{tabular}{|c|c|c|c|c|c|c|c|c|c|}
\hline Polymer & $\begin{array}{c}\text { Solvent } \\
\text { (Benzene/THF) }\end{array}$ & $\begin{array}{l}\text { Concentration } \\
(w / v[\%])\end{array}$ & $\begin{array}{l}\text { Phase separation } \\
\text { temperature }\left[{ }^{\circ} \mathrm{C}\right]\end{array}$ & $\begin{array}{l}\text { Density } \\
{\left[\mathrm{g} \mathrm{cm}^{-3}\right]}\end{array}$ & $\begin{array}{c}\text { Porosity } \\
{[\%]}\end{array}$ & $\begin{array}{l}\text { Pore Size } \\
\qquad[\mu \mathrm{m}]\end{array}$ & $\begin{array}{l}\text { Fiber Diameter } \\
{[\mathrm{nm}]}\end{array}$ & $\begin{array}{l}\text { Compressive } \\
\text { modulus [MPa] }\end{array}$ & $\begin{array}{c}\text { Compressive yield } \\
\text { strength [MPa] }\end{array}$ \\
\hline PLLA & $100 / 0$ & 7.5 & -20 & 0.125 & 90.1 & $120 \pm 16$ & - & 4.5 & 0.44 \\
\hline PLLA & $90 / 10$ & 7.5 & -20 & 0.102 & 91.9 & $107 \pm 15$ & $165 \pm 15$ & 4.2 & 0.41 \\
\hline PLLA & $80 / 20$ & 2.5 & -20 & 0.048 & 96.2 & $153 \pm 25$ & $158 \pm 20$ & 0.5 & 0.18 \\
\hline PLLA & $80 / 20$ & 5.0 & -20 & 0.065 & 94.8 & $126 \pm 18$ & $155 \pm 18$ & 2.9 & 0.30 \\
\hline PLLA & $80 / 20$ & 7.5 & -20 & 0.093 & 92.6 & $80 \pm 7$ & $157 \pm 21$ & 3.5 & 0.38 \\
\hline PLLA & $80 / 20$ & 10.0 & -20 & 0.110 & 91.3 & $77 \pm 9$ & $160 \pm 31$ & 6.4 & 0.52 \\
\hline PLLA & $80 / 20$ & 5.0 & -80 & 0.069 & 94.5 & $115 \pm 20$ & $158 \pm 20$ & 3.2 & 0.33 \\
\hline PLLA & $80 / 20$ & 5.0 & LN & 0.068 & 94.6 & $52 \pm 12$ & $154 \pm 23$ & 3.3 & 0.32 \\
\hline PLLA & $60: 40$ & 7.5 & -20 & 0.091 & 92.8 & $20 \pm 3$ & $143 \pm 20$ & 3.0 & 0.28 \\
\hline PLLA & $0 / 100$ & 7.5 & -20 & 0.094 & 92.5 & - & $144 \pm 22$ & 3.0 & 0.38 \\
\hline
\end{tabular}



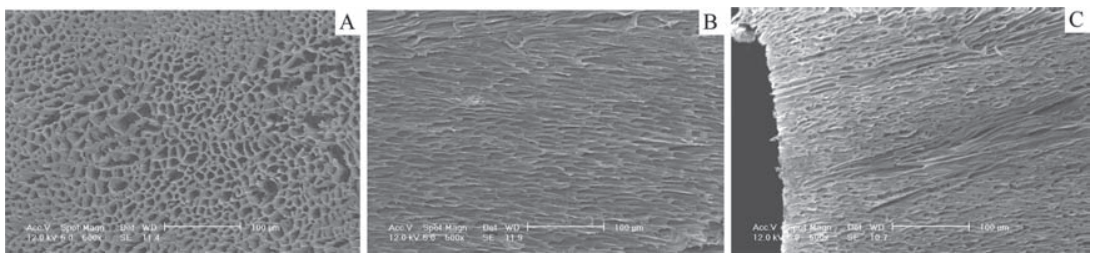

Figure 5. SEM images of the axially oriented gradient structure of scaffolds prepared using $5.0 \%(\mathrm{w} / \mathrm{v})$ PLLA/benzene solution at $-196{ }^{\circ} \mathrm{C}$ : A) cross section of tubular pore structure; B) cross section, $\mathrm{O} / \mathrm{I}$ structure; C) cross section, $1 / \mathrm{O}$ structure.

Compared to the simple solid-liquid phase separation occurring when using a PLLA/benzene solution, there are likely two stages of phase separation when using a PLLA/(benzene/THF) solution in the scaffold-fabrication process: a solid-liquid phase separation followed by a liquid-liquid phase separation. Benzene has a much higher freezing temperature than THF. Therefore, during the first stage of the solid-liquid phase separation, the crystallization of benzene creates micropores. The oriented gradient microtubular structure in the axial or radial direction (similar to Figure 2-5) could again be achieved during this step by utilizing a proper temperature gradient. After this stage, the polymer solution was separated into a solidified benzene phase and a polymer-rich PLLA/THF phase, which was partially compartmentalized by the benzene crystals. The nanofibrous structure was formed during the second stage by spinodal liquidliquid phase separation and subsequent crystallization of the polymer-rich phase. Figure $\mathbf{1 0}$ describes the proposed phaseseparation mechanism of PLLA/(benzene/THF) solutions.

\subsection{Mechanical Properties}

The mechanical properties, including the compressive modulus and compressive yield strength, of our scaffolds are shown in Tables 1 and 2. The typical results on the compressive yield strength are presented in Figure $\mathbf{1 1}$ and 12. For the scaffolds possessing oriented gradient microtubules, the anisotropic

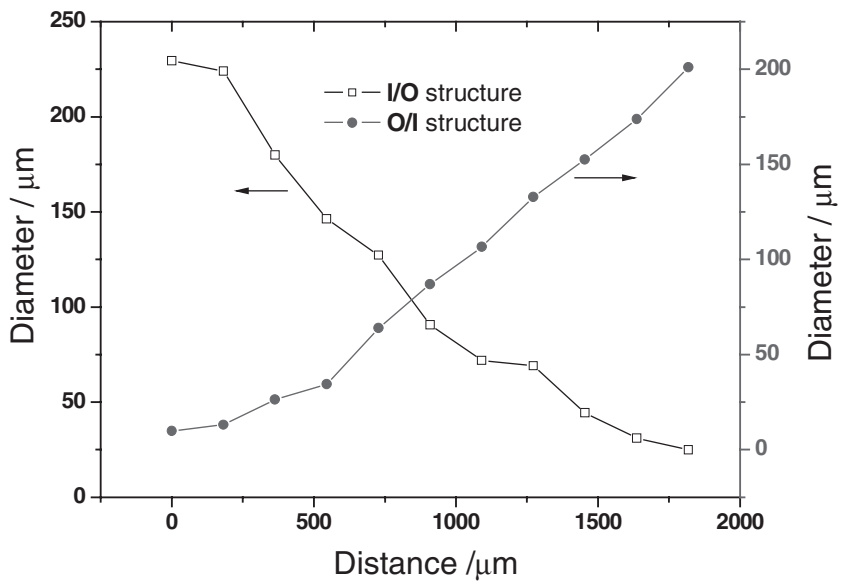

Figure 6. Pore size of the radially oriented gradient microtubular structure. (PLLA/benzene solutions with PLLA concentration of $5.0 \%$, phaseseparation temperature: $-20^{\circ} \mathrm{C}$ ) architecture led to anisotropic mechanical properties. Both the compressive modulus and the yield strength of scaffolds with a microtubular architecture were significantly greater in the longitudinal direction than in the transverse direction of the tubular structure (Table 1 and Figure 11). Both the compressive modulus and the compressive yield strength increased with polymer concentration, as expected (Tables 1 and 2 and Figure 11). At the same polymer concentration, there was no statistical difference in the mechanical properties between the two types of radially oriented gradient $(\mathrm{O} / \mathrm{I}$ or I/O) scaffolds (Table 1 and Figure 11).

The compressive modulus and compressive yield strength of the nanofibrous scaffolds with interconnected channels were both slightly lower than those of the scaffolds with a solid-walled oriented gradient structure at the same polymer concentration in the oriented direction of the microtubules. At similar polymer concentrations, the scaffolds with a solid-walled structure (prepared using benzene) had a larger average pore size and higher skeletal density of the pore walls, which may have led to the higher compressive modulus and yield strength. The average pore size became smaller and smaller as THF was introduced and continually increased, resulting in the looser aggregation of nanofibers in the pore walls, which might be associated with the lower compressive modulus and yield strength.

\subsection{Cellular Migration into the Scaffolds in Vivo}

To investigate cell migration into and distribution within the scaffolds of different pore structures in vivo, scaffolds with radially oriented and non-oriented pores were subcutaneously implanted into mice. After 2 weeks of implantation, haematoxylin and eosin (H-E) staining of the cross sections of the implants showed that abundant host cells migrated into the scaffolds with orientated pores and the fibroblast-like cells appeared to be healthy in the microchannels (Figure 13A). In contrast, substantially fewer cells migrated into the scaffolds with non-oriented pores (Figure 13B). Both types of scaffold maintained their geometrical shape and structural integrity during the 2-week implantation.

\section{Discussion}

It is well recognized that scaffolds play a critical role in tissue engineering. The three-dimensional pore structure and surface morphology of the scaffolds affect the quality of the tissue being developed on the scaffold. However, there are limited reports on scaffolds for blood-vessel engineering. Blood vessels, like many other tissues including nerve, muscle, tendon, ligament, bone and teeth, have oriented architectures.

In this paper, biodegradable PLLA scaffolds with an oriented microtubular structure along different directions were successfully created. We have demonstrated how to control the architectural parameters such as the porosity, tubular diameter, and orientational direction of the microtubules by varying the 

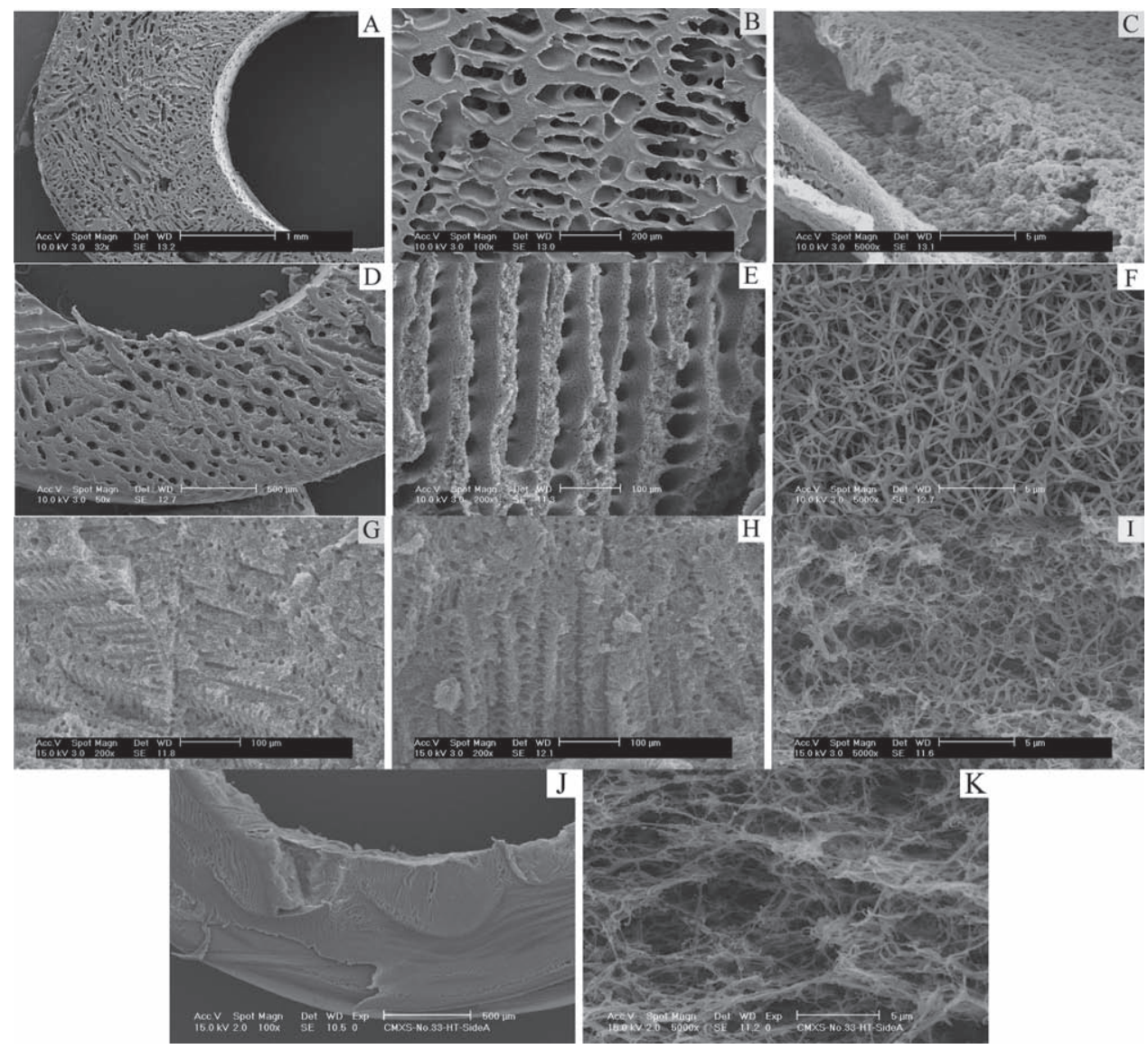

Figure 7. SEM images of PLLA scaffolds prepared using PLLA solutions in benzene and THF using the TIPS technique. A-C: $7.5 \%$ (w/v) PLLA/ (benzene/THF), benzene/THF (v/v) = 9:1; D-F: 7.5\% (w/v) PLLA/(benzene/THF), benzene/THF (v/v) = 8:2; G-l: 7.5\% (w/v) PLLA/(benzene/THF), benzene/THF $(\mathrm{v} / \mathrm{v})=6: 4 ;$ J-K: $7.5 \%$ (wt/v) PLLA/(benzene/THF), benzene/THF $(\mathrm{v} / \mathrm{v})=0: 10$. The TIPS temperature was $-20^{\circ} \mathrm{C}$.

processing parameters, such as the polymer concentration and temperature gradient. Moreover, by designing the molds using different materials for different parts, we created various oriented microtubules and gradient pore structures. For instance,

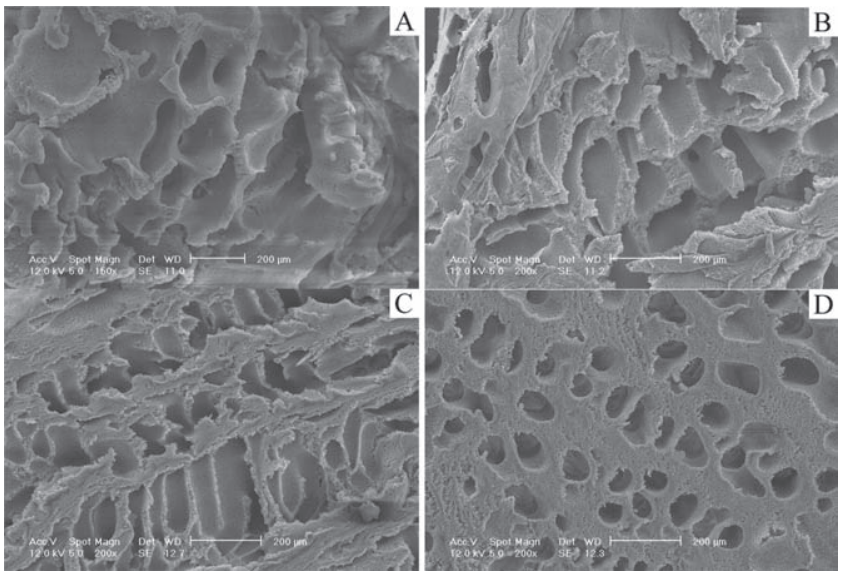

Figure 8. SEM images of PLLA scaffolds prepared with various polymer concentrations using the TIPS technique (benzene/THF $=8: 2$, PLLA concentration (w/v): A, 2.5\%; B: 5.0\%; C: 7.5\%; D: 10.0\%, the TIPS temperature was $\left.-20^{\circ} \mathrm{C}\right)$. a gradient scaffold was created with an oriented structure from the outside wall (with larger pores) to the inside wall (with smaller pores). The inside layer with smaller pores could be advantageous for the seeding and growth of endothelial cells (ECs), whereas the outside layer with the bigger pores could create a more suitable environment for the growth of smooth muscle cells (SMCs) and their matrix synthesis. It is also likely that the radially oriented microtubules within a scaffold provide an easier pathway for cell seeding and uniform distribution throughout the scaffold. The scaffold implantation study in this paper demonstrates that cells easily migrated into radially oriented tubular pores achieving a higher cell density and more uniform cell distribution throughout the scaffold than in the random pores of the control scaffold. The healthy appearance of the cells in the radially oriented pores may be related to the better mass-transfer conditions than those in the less interconnected pores of the control scaffold. The intact vessel-shaped geometry also demonstrates the adequate mechanical properties to support tissue regeneration in the ectopic implantation model used in this study. The effects and mechanisms of the pore structure and orientation on cell functions such as proliferation, differentiation, and new-tissue development along with the degradation of the polymer scaffolds in a blood-vessel replacement model will be studied in future investigation. 


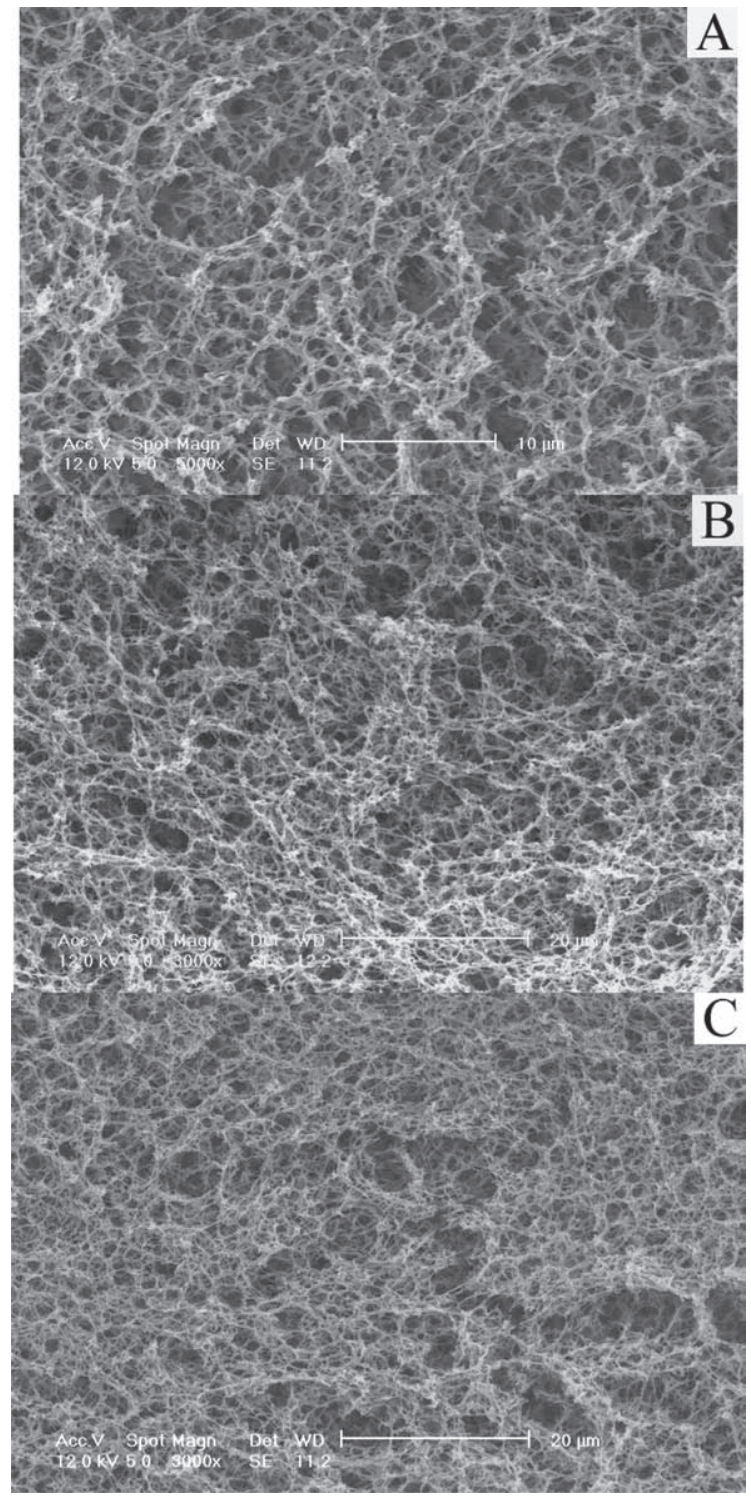

Figure 9. SEM images vs. the TIPS temperature (benzene/THF $=8: 2$, the PLLA concentration was $5.0 \%$ (wt/v). A: $\left.-20^{\circ} \mathrm{C} ; \mathrm{B}:-80^{\circ} \mathrm{C} ; \mathrm{C}:-196^{\circ} \mathrm{C}\right)$.

Inspired by the nanofibrous architecture of collagen in natural ECM, our laboratory previously developed synthetic nanofibrous polymer scaffolds with macro-/micropores by combining porogen template-leaching and phase-separation techniques. ${ }^{[6,38,44]}$ In the present work, we developed a technique to fabricate scaffolds with both interconnected microtubular pores and a nanofibrous pore-wall architecture without using any templates (preformed porogen materials). By simply controlling the ratio of benzene/THF, the polymer concentration, and the phase-separation temperature, scaffolds with different porosity, microtubule size, and nanofiber density on the microtubule walls can be created, allowing for the optimization of scaffolds for specific cells and specific tissues. Different from the non-directional spherical pore structure of the scaffolds generated by paraffin or sugar spheres, both oriented and interconnected microtubular networks could be achieved

\section{Homogeneous solution}

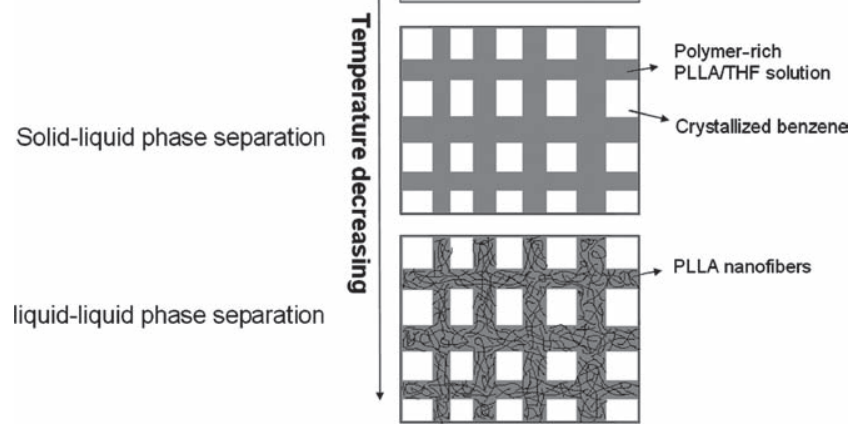

Figure 10. Schematic illustration of the nanofibrous scaffolds with interconnected microtubular structure fabricated using the TIPS process in PLLA/benzene/THF solutions.

in the scaffolds using the new methods developed in this work. The new fabrication technique is simple, tunable, and can also greatly shorten the production time of the scaffolds. Moreover, the technique is versatile and likely has a general applicability to other polymers as the mold design and the solvent type rather than the polymer type play the main roles in determining the gradient microtubular and nanofibrous architectures.

\section{Conclusions}

This paper presents a few types of novel scaffolds fabricated from biodegradable polymers by means of a new thermally induced phase-separation technique. Tubular biodegradable PLLA scaffolds were fabricated for the tissue engineering of small-diameter blood vessels by utilizing their nanostructural similarities to the ECM and advantageous pore design to

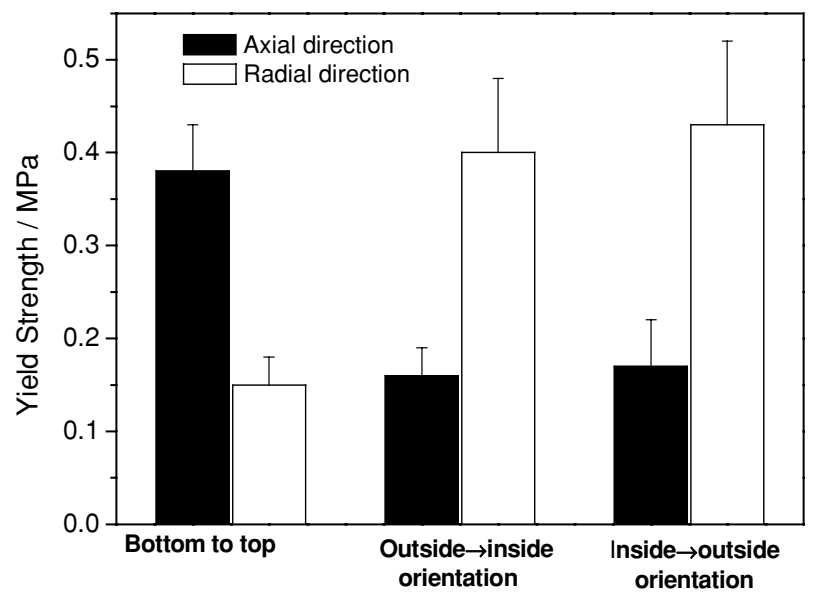

Figure 11. Compressive yield strength of PLLA scaffolds prepared with benzene as the solvent using the TIPS technique. The PLLA concentration was $5.0 \%$. The TIPS temperature was $-20^{\circ} \mathrm{C}$. 




Figure 12. Compressive yield strength of PLLA scaffolds prepared with PLLA solutions in benzene, THF, and a mixture of benzene/THF using the TIPS technique. The TIPS temperature was $-20^{\circ} \mathrm{C}$.

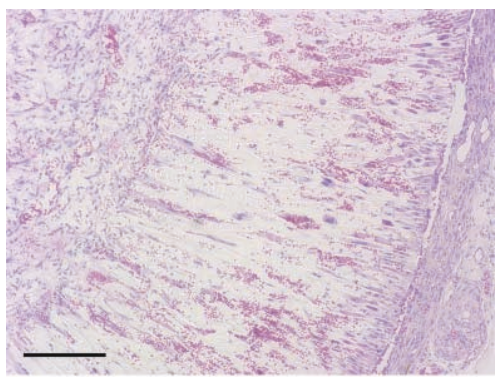

(A)



(B)

Figure 13. After 2 weeks of implantation, H-E staining of the cross sections of implants showed that abundant host cells migrated into the scaffolds with orientated pores and the fibroblast-like cells appeared to be healthy in the microchannels (Figure 13A), substantially fewer cells migrated into the scaffolds with non-oriented pores (Figure 13B). Both types of scaffold maintained their original shapes. Scale bar: $200 \mu \mathrm{m}$.

facilitate tissue regeneration. We have demonstrated that it is possible to control the architectural parameters such as the porosity, pore size, and the orientational direction of the microtubular pores by tuning the processing parameters, including the polymer concentration and temperature gradient, especially utilizing the differences in thermal conductivity of the mold materials. The gradient microtubular structure was shown to facilitate cell migration and uniform distribution throughout the scaffold in vivo. The oriented gradient pores were also intended to improve mass transfer conditions for cell growth and function. We also developed nanofibrous scaffolds with an oriented and interconnected microtubular pore network by a one-step TIPS method without the use of template materials.
The structural features of such scaffolds can be conveniently adjusted by varying the solvent ratio, phase-separation temperature, and polymer concentration. The fabrication technology does not require expensive facilities, is easy to carry out, and can shorten the scaffold fabrication time. These fabrication technologies are versatile and could be utilized to fabricate scaffolds and complex porous materials from different polymers for tissue engineering and various other applications.

\section{Experimental Section}

Materials: PLLA with an inherent viscosity of 1.4-1.8 $\mathrm{dl} \mathrm{g}^{-1}$ was purchased from Boehringer Ingelheim (Ingelheim, Germany) and was used as received. Benzene, THF, and other reagents were obtained from Aldrich Chemical Company (Milwaukee, WI). They were of analytical grade and used without further treatment.

Vessel Scaffold Fabrication: Three different molds were designed to fabricate different blood-vessel scaffolds with orientation and gradient pore structures. The molds were composed of top and bottom plates, an inner shaft, and an outer cylinder made of the same or different materials, as shown in Figure 1. The usage of different materials (metal and Teflon) was intended to create different temperature gradients for the TIPS process. In the present study, molds with an inner-diameter of $3.00 \mathrm{~mm}$ and an outer-diameter of $5.00 \mathrm{~mm}$ were used.

The PLLA was dissolved in benzene to form a homogeneous solution with a concentration in the range of $2.5 \%-10 \%$. The polymer solution was poured into different molds then transferred into a freezer set to a chosen temperature to induce phase separation. The phase-separated polymer/solvent system was then transferred into a freeze drying vessel at -5 to $-10{ }^{\circ} \mathrm{C}$ in an ice/salt bath, and was freeze-dried under vacuum (pressure lower than $0.5 \mathrm{~mm} \mathrm{Hg}$ ) for $72 \mathrm{~h}$. The dried scaffolds were then kept in a desiccator until characterization or usage.

For the preparation of nanofibrous PLLA scaffolds, a mixture of benzene and THF with various ratios was used as the solvent. After phase separation, the molds containing the polymer solution were immersed into cold hexane for 2 days to extract the solvents, changing the hexane three times a day. Hexane was then exchanged with cyclohexane. The polymer scaffolds were removed from the cyclohexane, and frozen at $-70{ }^{\circ} \mathrm{C}$ for at least $5 \mathrm{~h}$. The frozen scaffolds were lyophilized at $-10{ }^{\circ} \mathrm{C}$ for $72 \mathrm{~h}$ and then kept in a desiccator until usage.

Structure/Property Characterization: To estimate the density and porosity of the PLLA scaffolds, the inner-diameter, outer-diameter, and height of each scaffold were measured after freeze-drying to calculate the volume of each scaffold. The mass of each scaffold was measured with an analytical balance, and the overall density $\left(D_{f}\right)$ was calculated from the volume and the mass. The porosity $\varepsilon$ of each scaffold was calculated from the measured overall density $D_{f}$ of the fibrous matrix and the skeletal density $D_{\mathrm{p}}$ using previously described techniques. The porosity was defined as:

$\varepsilon=\frac{D_{\mathrm{p}}-D_{\mathrm{f}}}{D_{\mathrm{p}}}$

where the skeletal density $D_{p}$ of the scaffolds was given by:

$D_{p}=\frac{1}{\frac{1-X_{c}}{D_{a}}+\frac{X_{c}}{D_{c}}}$

where $X_{c}$ is the degree of crystallinity determined by differential scanning calorimetry as described elsewhere. ${ }^{[36]}$ For PLLA, $D_{\mathrm{a}}=1.248 \mathrm{~g} \mathrm{~mL}^{-1}$ (density of amorphous polymer) and $D_{c}=1.290 \mathrm{~g} \mathrm{~mL}^{-1}$ (density of $100 \%$ crystalline polymer).

The porous morphologies of the scaffolds were examined using scanning electron microscopy (SEM) (S-3200N, Hitachi, Japan). To expose the internal architecture, a sample was cut carefully with a razor 
blade after freeze-drying. All samples were coated with gold using a sputter coater (Desk-II, Denton Vacuum Inc., Moorstown NJ) with a pressure below $50 \mathrm{mTorr}$, a current of approximately $40 \mathrm{~mA}$, and a coating time of $120 \mathrm{~s}$. The diameters of the pores and the nanofibers were measured from SEM images using Image-pro plus software (Media Cybernetics). More than 40 micropores and nanofibers were chosen to calculate an average diameter. To determine the gradient structure of the pores, each wall thickness was divided into ten equal parts from the inner-wall to the outer-wall or from outer-wall to inner-wall.

The compressive mechanical properties of the PLLA scaffolds were measured with an MTS Synergie 200 mechanical tester (MTS Systems Corporation, Eden Prairie, MN). For compression testing, the specimens were homocentric tubes measuring $5 \mathrm{~mm}$ in outer-diameter, $3 \mathrm{~mm}$ in inner-diameter, and $3.0 \mathrm{~mm}$ in height. The load was applied in the direction either parallel or perpendicular to the tubular axis. The crosshead speed was $0.5 \mathrm{~mm} \mathrm{~min}{ }^{-1}$ and the compressive modulus was defined as the initial linear modulus. The yield strength was determined from the cross point of the two tangents on the stress-strain curve around the yield point. At least 5 specimens were tested for each sample.

Subcutaneous Implantation: The animal procedures were performed according to the protocol approved by the University of Michigan Committee of Use and Care of Laboratory Animals. The tubular scaffolds (2 $\mathrm{mm}$ in thickness) were implanted into subcutaneous pockets of 6-8 weeks old C57BL/6 male mice (Charles River Laboratories, Wilmington, MA). Surgery was performed under general inhalation anesthesia with isofluorane. Two midsagittal incisions were made on the dorsa and one subcutaneous pocket was created on each side of the incisions using blunt dissection. One scaffold was implanted subcutaneously into each pocket. Four samples were implanted for each group. After placement of implants, the incisions were closed with staples. At the end of the 2-week implantation period, the mice were euthanized and the implants were harvested.

Histological Analysis: Implants were washed in phosphate-buffered saline (PBS), fixed with 3.7\% formaldehyde in PBS overnight, dehydrated through a graded series of ethanol, embedded in paraffin, and sectioned at a thickness of $5 \mu \mathrm{m}$. The sections were deparaffinized, rehydrated with a graded series of ethanol, and stained with hematoxylin and eosin (H-E).

\section{Acknowledgements}

The research was supported by the National Institutes of Health (NIDCR Research Grants DE015384 and DE017689: PXM).

Received: May 9, 2010 Published online: July 22, 2010

[1] B. C. Isenberg, C. Williams, R. T. Tranquillo, Circulation Res. 2006, 98, 25 .

[2] R. M. Nerem, D. Seliktar, Annu. Rev. Biomed. Eng. 2001, 3, 225.

[3] L. Germain, M. Remy-Zolghadri, F. Auger, Med. Biol. Eng. Comp. 2000, 38, 232.

[4] O. E. Teebken, A. Haverich, Eur. J. Vasc. Endovasc. Surg. 2002, 23, 475.

[5] P. X. Ma, Adv. Drug Delivery Rev. 2008, 60, 184.

[6] G. B. Wei, P. X. Ma, Adv. Funct. Mater. 2008, 18, 3568.

[7] Y. Q. Wang, D. E. Noga, K. Yoon, A. M. Wojtowicz, A. S. P. Lin, A. J. Garcia, D. M. Collard, M. Weck, Adv. Funct. Mater. 2008, 18, 3638.

[8] Y. J. Zhang, S. P. Wang, M. Eghtedari, M. Motamedi, N. A. Kotov, Adv. Funct. Mater. 2005, 15, 725 .

[9] A. R. Boccaccini, F. Chicatun, J. Cho, O. Bretcanu, J. A. Roether, S. Novak, Q. Chen, Adv. Funct. Mater. 2007, 17, 2815.
[10] B. J. Xie, R. L. Parkhill, W. L. Warren, J. E. Smay, Adv. Funct. Mater. 2006, 16, 1685.

[11] R. Zhang, P. X. Ma, J. Biomed. Mater. Res. 1999, 44, 446.

[12] T. Shinoka, P. X. Ma, D. Shum-Tim, C. K. Breuer, R. A. Cusick, G. Zund, R. Langer, J. P. Vacanti, J. E. Mayer, Jr., Circulation 1996, 94, 11164.

[13] T. Shinoka, D. Shum-Tim, P. X. Ma, R. E. Tanel, N. Isogai, R. Langer J. P. Vacanti, J. E. Mayer, J. Thorac. Cardiovasc. Surg. 1998, 115, 536.

[14] T. Shinoka, C. K. Breuer, R. E. Tanel, G. Zund, T. Miura, P. X. Ma R. Langer, J. P. Vacanti, J. E. Mayer, Jr., Ann. Thorac. Surg. 1995, 60, S513.

[15] C. K. Breuer, T. Shinoka, G. Zund, D. J. Mooney, P. X. Ma, R. Langer, T. E. Mayer, J. P. Vacanti, Biotechnol. Bioeng. 1996, 50, 562.

[16] J. P. Stegemann, S. N. Kaszuba, S. L. Rowe, Tissue Eng. 2007, 13 2601.

[17] C. E. Sarraf, A. B. Harris, A. D. McCulloch, M. Eastwood, Cell Proliferation 2003, 36, 241

[18] H. B. He, T. Matsuda, Tissue Eng. 2002, 8, 213.

[19] E. D. Boland, J. A. Matthews, K. J. Pawlowski, D. G. Simpson, G. E. Wnek, G. L. Bowlin, Front. Biosci. 2004, 9, 1422.

[20] F. Opitz, K. Schenke-Layland, W. Richter, D. P. Martin, I. Degenkolbe, T. Wahlers, U. A. Stock, Ann. Biomed. Eng. 2004, 32, 212.

[21] C. Y. Xu, R. Inai, M. Kotaki, S. Ramakrishna, Tissue Eng. 2004, 10, 1160.

[22] V. Mironov, V. Kasyanov, X. Z. Shu, C. Eisenberg, L. Eisenberg, S. Gonda, T. Trusk, R. R. Markwald, G. D. Prestwich, Biomaterials 2005, 26, 7628

[23] P. Engbers-Buijtenhuijs, L. Buttafoco, A. A. Poot, P. J. Dijkstra, R. A. I. de Vos, L. M. T. Sterk, R. H. Geelkerken, I. Vermes, J. Feijen, Biomaterials 2006, 27, 2390.

[24] S. Sarkar, G. Y. Lee, J. Y. Wong, T. A. Desai, Biomaterials 2006, 27, 4775 .

[25] J. Stitzel, L. Liu, S. J. Lee, M. Komura, J. Berry, S. Soker, G. Lim, M. Van Dyke, R. Czerw, J. J. Yoo, A. Atala, Biomaterials 2006, 27 1088.

[26] G. R. Campbell, J. H. Campbell, Curr. Pharmac. Biotechnol. 2007, 8, 43.

[27] F. Couet, N. Rajan, D. Mantovani, Macromol. Biosci. 2007, 7, 701.

[28] M. Lovett, C. Cannizzaro, L. Daheron, B. Messmer, G. VunjakNovakovic, D. L. Kaplan, Biomaterials 2007, 28, 5271.

[29] J. J. Stankus, L. Soletti, K. Fujimoto, Y. Hong, D. A. Vorp, W. R. Wagner, Biomaterials 2007, 28, 2738.

[30] L. E. Niklason, J. Gao, W. M. Abbott, K. K. Hirschi, S. Houser R. Marini, R. Langer, Science 1999, 284, 489.

[31] P. Zorlutuna, N. Hasirci, V. Hasirci, J. Tissue Eng. Regen. Med. 2008 2, 373.

[32] J. Yang, D. Motlagh, A. R. Webb, G. A. Ameer, Tissue Eng. 2005, 11 1876.

[33] P. X. Ma, R. Y. Zhang, J. Biomed. Mater. Res. 2001, 56, 469.

[34] X. X. Hu, H. Shen, F. Yang, J. Z. Bei, S. G. Wang, Biomaterials 2008, $29,3128$.

[35] C. M. Vaz, S. van Tuijl, C. V. C. Bouten, F. P. T. Baaijens, Acta Biomater. 2005, 1, 575

[36] P. X. Ma, R. Y. Zhang, J. Biomed. Mater. Res. 1999, 46, 60.

[37] V. J. Chen, P. X. Ma, Biomaterials 2004, 25, 2065.

[38] G. B. Wei, P. X. Ma, J. Biomed. Mater. Res. Part A 2006, 78A, 306.

[39] K. M. Woo, V. J. Chen, P. X. Ma, J. Biomed. Mater. Res. Part A 2003, 67A, 531.

[40] K. M. Woo, J. H. Jun, V. J. Chen, J. Y. Seo, J. H. Baek, H. M. Ryoo, G. S. Kim, M. J. Somerman, P. X. Ma, Biomaterials 2007, 28, 335.

[41] V. J. Chen, L. A. Smith, P. X. Ma, Biomaterials 2006, 27, 3973.

[42] J. Hu, K. Feng, X. Liu, P. X. Ma, Biomaterials 2009, 30, 5061.

[43] J. Hu, X. Liu, P. X. Ma, Biomaterials 2008, 29, 3815.

[44] P. X. Ma, J. W. Choi, Tissue Eng. 2001, 7, 23. 